\title{
ANÁLISE DA ATIVIDADE HORMONAL DE TIAMETOXAM ATRAVÉS DE BIOTESTES
}

\author{
Paulo R. C. Castro ${ }^{1}$, Angélica M. C. M. Pitelli ${ }^{2}$, Lázaro E. P. Peres ${ }^{1}$, Paulo H. Aramaki ${ }^{3}$
}

\author{
${ }^{1}$ Departamento de Ciências Biológicas, ESALQ/USP, Av. Pádua Dias, 11, Piracicaba, SP. C.P.: 9, CEP: 13.418-900, \\ E-mail: prcastro@esalq.usp.br \\ ${ }^{2}$ CPG Fisiologia e Bioquímica de Plantas, ESALQ/USP. \\ ${ }^{3}$ Syngenta Proteção de Cultivos Ltda.
}

\section{RESUMO}

Com finalidade de determinar se a molécula de tiametoxam se caracteriza como um regulador vegetal (biorregulador) pertencente ao grupo das giberelinas, auxinas ou citocininas (promotores de crescimento), foram realizados biotestes em gerbox, sob condições controladas de laboratório com plantas-teste de tomateiro 'Micro-Tom' e com seus mutantes DGT (diagetrópico) insensível para auxina e BRT (bushy root) auxina/ citocinina, de raízes ramificadas. Como fonte de tiametoxam foi utilizado $30 \mathrm{ml}$ do produto Cruiser $35 \mathrm{FS}$ embebido em papel filtro nos gerbox nas concentrações de 0,$0 ; 0,1 ; 1,0 ; 10,0 ; 100,0$ e $1000,0 \mu \mathrm{M}$. Foram semeadas 10 sementes desinfectadas em cada gerbox, utilizando-se 10 repetições. O comprimento da raiz e da parte aérea das plântulas foram determinados após 4 dias. As médias foram colocadas em gráficos avaliando-se o desvio padrão. Os resultados mostraram que a molécula de tiametoxam não possui atividade de giberelina, de auxina ou de citocinina.

Palavras-chave: Lycopersicon esculentum, mutantes hormonais, Cruiser.

\section{ANALYSIS OF HORMONAL ACTIVITY OF THIAMETOXAN TROUGHT BIOTESTS}

\begin{abstract}
The present work aimed to study the possible hormonal activity of thiametoxan using biotests with 'Micro-Ton' (MT) dwarf tomato and its hormonal mutants DGT (auxin insensitivy) and BRT (bushy root). Germinated seeds of MT and mutants DGT and BRT were placed in a black Ger-Box containing water (control) and thiametoxan $0.1 ; 1.0 ; 10.0 ; 100.0$ and $1000.0 \mu \mathrm{M}$. After 4 days the lenght of roots and hypocotyls were measured. The promotion of hypocotyls growth did not occur in MT. In the mutant DGT differences were not observed. Mutant BRT did not answer in growth of roots and hypocotyls under effect of thiametoxan. By these results it can be concluded that thiametoxan did not act as a gibberellin, an auxin or a cytokinin.
\end{abstract}

Key words: Lycopersicon esculentum, mutants, Cruiser 


\section{INTRODUÇÃO}

A soja se consolidou como a principal cultura do agronegócio brasileiro, a partir da década de 70 , passando de 1,5 milhões de toneladas (1970) para mais de 15 milhões de toneladas (1979). Esse crescimento foi devido não apenas à expansão da área plantada, como também, ao expressivo aumento da produtividade, em função das novas tecnologias que foram disponibilizadas aos produtores (EMBRAPA, 2002).

A cultura da soja está sujeita durante todo o seu ciclo, ao ataque de diferentes espécies de insetos. Vários trabalhos encontrados na literatura têm demonstrado eficácia do tiametoxam no controle de insetos-praga, resultando em aumentos de produção (Siddiqui \& Trimohan, 2000; Avila \& Gomes, 2003; Proft et al., 1999; Seculik et al., 1999; Hrncic \& Jakic, 1999; Liguori et al., 2002). Visando controle das pragas iniciais, medidas como o tratamento de sementes, têm se destacado pelas vantagens como: aproveitamento dos equipamentos de plantio, fácil manuseio e economia de custos adicionais e também para não se afetar certos inimigos naturais como o parasitóide Cotesia arginiventris (Tillman \& Scott, 1997; Rao et al., 2003). O fechamento mais rápido da cultura também é desejável, pois contribui para o controle das plantas invasoras (EMBRAPA, 2002). Desta forma, Avila \& Gomes (2003) observaram ganho de produção em soja com aplicação de tiametoxam ( $98 \mathrm{~g}$ i.a ha ${ }^{-1}$ ) via tratamento de sementes. Trabalhos de investigação sobre possíveis efeitos fisiológicos deste produto sobre o desenvolvimento das plantas de soja não foram encontrados.

Com o objetivo de verificar se a molécula de tiametoxam se caracteriza como um regulador vegetal foram realizados biotestes com tomateiro 'Micro-Tom', DGT e BRT, de acordo com as técnicas preconizadas por Scott (1990) e Reid (1993).

\section{MATERIAL E MÉTODOS}

As sementes de tomateiro foram desinfectadas em solução $5 \%$ de hipoclorito por 5 minutos e depois lavadas abundantemente com água destilada. Foram colocadas 10 sementes em cada Gerbox, sendo que foram feitas 2 caixas para cada tratamento para que se obtivesse no mínimo 10 plantas por tratamento (Pitelli, 2006).

Os genótipos utilizados foram: (a) MT, cultivar anão do tomateiro; (b) DGT (diageotropico), mutante insensível a auxina; e (c) BRT (bushy root), mutante IAA/ citocinina, raízes ramificadas. Esses genótipos foram utilizados com a finalidade de verificar a resposta no desenvolvimento da radícula e do hipocótilo dos mesmos ao tiametoxam $(3-(2$ - cloro - tiazol - 5 ilmetil $)-5-$ metil - $(1,3,5)$ oxadiazinan -4 ilideno $-\mathrm{N}$ - nitroamina).

Sabe-se que MT, o cultivar anão de tomateiro, responde a substâncias do grupo das giberelinas apresentando crescimento do hipocótilo e restrição no crescimento da radícula. O mutante DGT (diageotrópico) é uma planta anã com crescimento diageotrópico dos caules e raízes, com raízes sem ramificação e defectivo na via de transdução do sinal de auxina, respondendo a substâncias desse grupo. O mutante BRT (raízes ramificadas) é uma planta anã que apresenta raízes excessivamente ramificadas, possuindo sensibilidade para citocininas (IAA/ Cks).

Foram colocados $30 \mathrm{ml}$ das soluções de tiametoxam 0,$0 ; 0,1 ; 1,0 ; 10,0 ; 100,0$ e $1000,0 \mu \mathrm{M}$ no papel filtro de cada gerbox. Após 4 dias foram avaliados o comprimento da raiz e da parte aérea das plântulas. As médias foram colocadas em gráficos nos quais também se avaliou o erro padrão de cada tratamento. 


\section{RESULTADOS E DISCUSSÃO}

\section{MT}

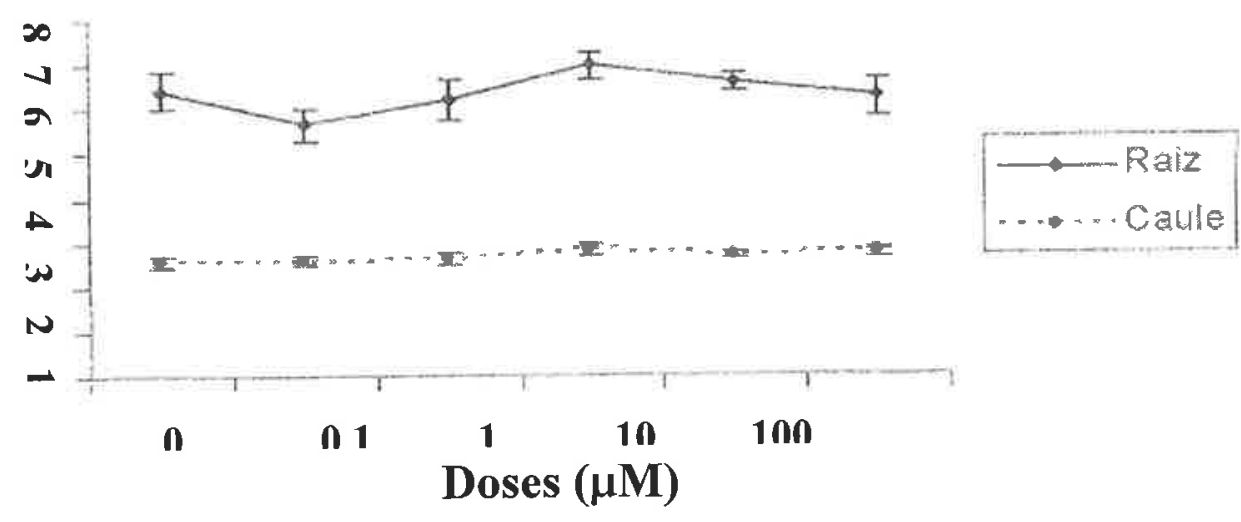

Figura 1. Efeito de diferentes concentrações de tiametoxam no comprimento de radículas e hipocótilos de tomateiro cultivar Micro-Tom.

\section{DG}

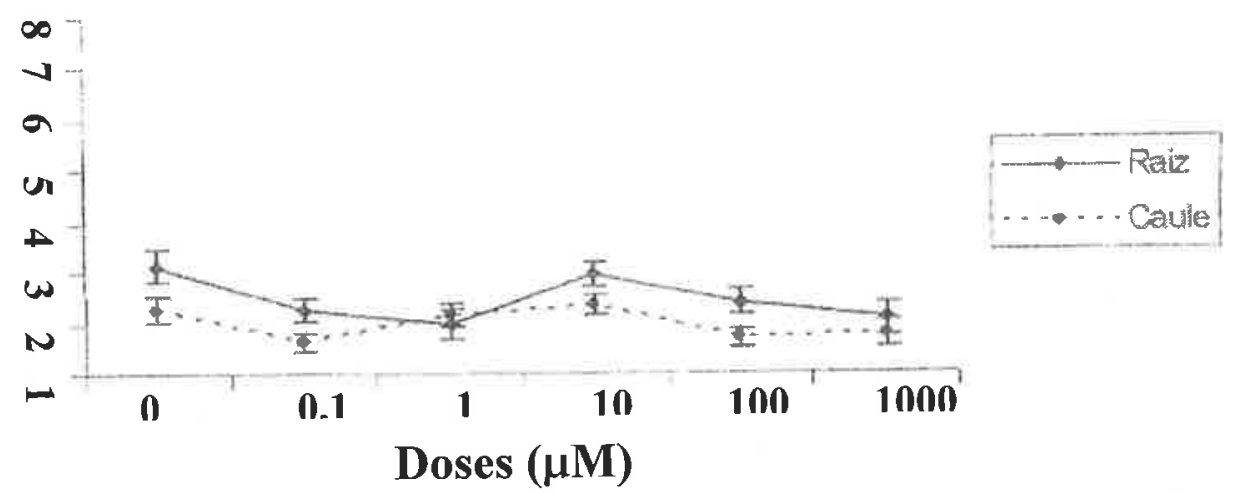

Figura 2. Efeito de diferentes concentrações de tiametoxam no comprimento de radículas e hipocótilos de tomateiro mutante DGT.

\section{BR}

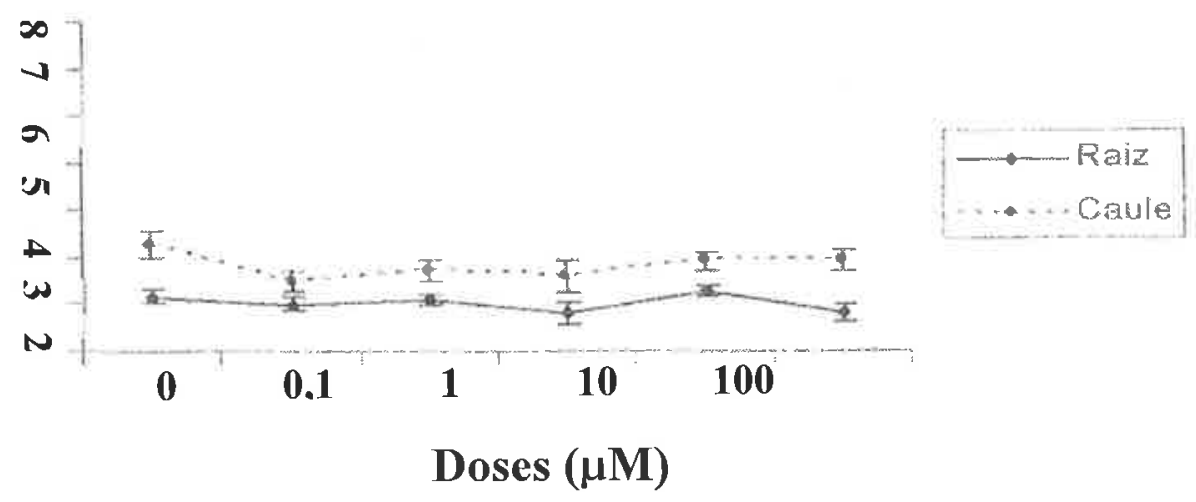

Figura 3. Efeito de diferentes concentrações de tiametoxam no comprimento de radículas e hipocótilos de tomateiro mutante BRT. 
De acordo com os dados obtidos e os gráficos apresentados, nos limites das concentrações utilizadas, o desenvolvimento da parte aérea e do sistema radicular das plântulas de tomateiro MT, DGT e BRT não demonstraram efeito de giberelina, auxina ou citocinina produzido pela molécula de tiametoxam.

\section{CONCLUSÕES}

Tiametoxam não afetou o desenvolvimento da parte aérea e do sistema radicular das plântulas teste de tomateiro.

A molécula não apresentou ação de giberelina, auxina ou de citocinina, de acordo com os biotestes aplicados.

\section{REFERÊNCIAS BIBLIOGRÁFICAS}

AVILLA, C.J.; GOMES, S.A. 2003. Effects of insecticides applied on seeds and in sowing furrows in the presence of the soybean white grub, Phyllophaga cuyabana. Embrapa, Centro de Pesquisa Agropecuária do Oeste. (Documentos, n. 55), 27 p.

EMBRAPA. 2002. Tecnologias de produção de soja. Sistemas de Produção 1, 199 p.

HRNCIC, S.; JAKIC, O. 1999. Efficiency of some new insecticides in controlling Colorado beetle. Poljoprivreda I Sumarstvo - Agriculture and Forestry, v. 45, n. 1-2, p. 85-90.

LIGUORI, R.; BERTONA, A.; MERLANO, M.; CASOLA, F.; BASSI, R.; FILI, V. 2003. Actara. Second generation neonicotinoid base on the new active ingredient thiamethoxan (Fruit crops vegetable crops - ornamental plants). Atti delle Giornate Fitopatologiche, n. 1, p. $341-346$.

PITELLI, A. M. C. M. 2006. Controle hormonal do crescimento de raízes de tomateiro (Lycopersicon esculentum $\mathrm{cv}$. Micro-Tom em condições de déficit hídrico. (Dissertação de Mestrado). Escola Superior de Agricultura "Luiz de Queiroz", USP, Piracicaba, 54 p.

PROFT, M.; RYCKEL, B.; DUCAT, N.; PIGEON, O.; BERNES, A. 1999. Pest control of sugar beet (Beta vulgaris), maize (Zea mays) and cereal crops by seed treatment with Thiamethoxan, Mededelingen Faculteit Landbouwkundige em Toegepaste Biologische, v. 64, n. 3a., p. $327-341$.

RAO, K. R.; PATHAK, K. A.; SHYLESHA, A. N. 2003. Influence of different formulations of insecticides on insect pests and their natural enemies of soybean. Indian Journal of Plant Protection, v. 31 n.1, p. $129-133$.

REID, J. B. 1993. Plant hormone mutants. Journal Plant Growth Regulation, v. 12, p. $207-226$.

SCOTT, I. M. 1990. Plant hormone response mutants. Physiologia Plantarum, v. 78, p. $147-152$.

SECULIK, R.; MASIREVIC, S.; KERESI, T. 1999. Possibilities for the control of harmful organisms in sunflower by treating the seed with insecticides. Zbornic Radova Naucni Institute za Ratarstvo I Povrtarstvo, n. 31, p. 467 $-478$.

SIDDIQUI, K. H.; TRIMOHAN. 2000. Evaluation of some insecticidal formulations against major insect pests (Melanagromyza sojae Zehnt.). Shashpa, v. 7, n.2, p. $167-170$.

TILLMAN, P. G.; SCOOT, W. 1997. Susceptibility of Cotesia marginoventris (Cresson) (Hymenoptera: Braconidae) to field rates of selected cotton insecticides. Journal of Entomological Science, v. 32, n. 3, p. $303-310$. 